PROCEEDINGS OF THE

AMERICAN MATHEMATICAL SOCIETY

Volume 134, Number 1, Pages 265-272

S 0002-9939(05)08040-8

Article electronically published on August 19, 2005

\title{
TOPOLOGICAL INVARIANCE OF THE SIGN OF THE LYAPUNOV EXPONENTS IN ONE-DIMENSIONAL MAPS
}

\author{
HENK BRUIN AND STEFANO LUZZATTO \\ (Communicated by Michael Handel)
}

\begin{abstract}
We explore some properties of Lyapunov exponents of measures preserved by smooth maps of the interval, and study the behaviour of the Lyapunov exponents under topological conjugacy.
\end{abstract}

\section{Statement of RESUlts}

In this paper we consider $C^{3}$ interval maps $f: I \rightarrow I$, where $I$ is a compact interval. We let $\mathcal{C}$ denote the set of critical points of $f: c \in \mathcal{C} \Leftrightarrow D f(c)=0$. We shall always suppose that $\mathcal{C}$ is finite and that each critical point is non-flat: for each $c \in \mathcal{C}$, there exist $\ell=\ell(c) \in[2, \infty)$ and $K$ such that $\frac{1}{K} \leq \frac{|f(x)-f(c)|}{|x-c|^{\ell}} \leq K$ for all $x \neq c$. Let $\mathcal{M}$ be the set of ergodic Borel $f$-invariant probability measures. For every $\mu \in \mathcal{M}$, we define the Lyapunov exponent $\lambda(\mu)$ by

$$
\lambda(\mu)=\int \log |D f| d \mu .
$$

Note that $\int \log |D f| d \mu<+\infty$ is automatic since $D f$ is bounded. However we can have $\int \log |D f| d \mu=-\infty$ if $c \in \mathcal{C}$ is a fixed point and $\mu$ is the Dirac- $\delta$ measure on c. It follows from [15, 11 that this is essentially the only way in which $\log |D f|$ can be non-integrable: if $\mu(\mathcal{C})=0$, then $\int \log |D f| d \mu>-\infty$.

The sign, more than the actual value, of the Lyapunov exponent can have significant implications for the dynamics. A positive Lyapunov exponent, for example, indicates sensitivity to initial conditions and thus "chaotic" dynamics of some kind. Our main result concerns the extent to which the sign of the Lyapunov exponent, which is a priori a purely metric condition, is in fact intrinsically constrained by the topological structure of the dynamics.

Theorem 1. If $f$ is $C^{3}$ with finitely many non-flat critical points, and if $\mu$ is non-atomic, then the sign of $\lambda(\mu)$ is a topological invariant.

We recall that $\mu$ is non-atomic if every point has zero measure. By the statement that the sign of $\lambda(\mu)$ is a topological invariant we mean the following. Two maps $f: I \rightarrow I$ and $g: J \rightarrow J$ are topologically conjugate if there exists a homeomorphism

Received by the editors September 5, 2004.

2000 Mathematics Subject Classification. Primary 37B10; Secondary 37A35, 11K99, 37A45.

The authors thank Juan Rivera-Letelier for drawing their attention to previous results relating to Lemma 1 and Proposition 1. They also thank Feliks Przytycki for pointing out an error in an earlier version of this paper.

(C)2005 American Mathematical Society Reverts to public domain 28 years from publication 
$h: I \rightarrow J$ such that $h \circ f=g \circ h$. The conjugacy $h$ induces a bijection between the space of ergodic invariant probability measures of $f$ and of $g$ : if $\mu_{f}$ is an ergodic invariant probability measure for $f$, then the corresponding measure $\mu_{g}$, defined by $\mu_{g}(A)=\mu_{f}\left(h^{-1}(A)\right)$ for all measurable sets $A$, is an ergodic invariant probability measure for $g$. Theorem 1 says that as long as both $f$ and $g$ are $C^{3}$ with finitely many non-flat critical points and $\mu_{f}$ is non-atomic, then the Lyapunov exponents $\lambda\left(\mu_{f}\right)$ and $\lambda\left(\mu_{g}\right)$ have the same sign. Clearly the actual values can vary.

The non-atomic condition is necessary in general, as a topological conjugacy can easily map a hyperbolic attracting/repelling periodic point to a topologically attracting/repelling ${ }^{1}$ neutral periodic point. The corresponding Lyapunov exponents of the corresponding Dirac- $\delta$ measures would then be positive and zero, respectively. The result is concerned with the more interesting non-atomic case and in particular shows that the property that the exponent is zero or positive is topologically invariant (we shall show below that the negative Lyapunov exponent case always corresponds to an atomic measure).

The integrability of $\log |D f|$ means that our definition of Lyapunov exponents, commonly used in the one-dimensional context, agrees with the more classical definition in terms of the limit of the rate of growth of the derivative. Indeed, a standard application of Birkhoff's ergodic theorem (which relies on the integrability property) gives

$$
\lim _{n \rightarrow \infty} \frac{1}{n} \log \left|D f^{n}(x)\right|=\int \log |D f| d \mu=\lambda(\mu) \quad \text { for } \mu \text {-a.e. } x \text {. }
$$

This pointwise definition can be generalised to the so-called upper and lower Lyapunov exponents

$$
\lambda_{-}(x):=\liminf _{n \rightarrow \infty} \frac{1}{n} \log \left|D f^{n}(x)\right| \quad \text { and } \quad \lambda_{+}(x):=\limsup _{n \rightarrow \infty} \frac{1}{n} \log \left|D f^{n}(x)\right| .
$$

These quantities are defined at every point, and a natural generalisation of the question answered above is whether the signs of these upper and lower Lyapunov exponents are topological invariants. It was shown in [13] in the unimodal setting, that the positivity of the lower Lyapunov exponent along the critical orbit (the Collet-Eckmann condition) is preserved under topological conjugacy. This result does not hold for multimodal maps (see [16]) although it does generalise under additional recurrence conditions on the critical orbits [10]. In [16] it is also shown that in the context of rational maps on the Riemann sphere, the property that the Lyapunov exponents of all invariant measures are uniformly positive is preserved under topological conjugacy. It is not known whether this extends to $C^{2}$ interval maps.

If $f$ is unimodal and Collet-Eckmann, then every point has a positive upper Lyapunov exponent 14. As the Collet-Eckmann condition is preserved under conjugacy, the sign of upper pointwise Lyapunov exponent is preserved under conjugacy for Collet-Eckmann maps. However we show that at least for lower Lyapunov exponents this is false in general.

Proposition 1. There exist unimodal maps with points for which the sign of the lower pointwise Lyapunov exponent is not preserved under topological conjugacy.

\footnotetext{
${ }^{1}$ If $f$ has negative Schwarzian derivative, then a neutral periodic point cannot be two-sided repelling.
} 
This is not restricted to orbits asymptotic to neutrally attracting or neutrally repelling periodic orbits.

In [16] this result was proved for bimodal maps; their argument would not apply to the unimodal case, but shows that the lower pointwise Lyapunov exponent need not be preserved under a quasi-symmetric conjugacy.

We make the following conjecture:

Conjecture 1. Topological conjugacy preserves the sign of the upper pointwise Lyapunov exponents of all points that are not attracted to a periodic orbit.

It is immediate from the ergodic theorem that for every invariant measure $\mu$, there are points $x$ such that the Lyapunov exponent $\lambda(\mu)$ coincides with the pointwise Lyapunov exponent $\lambda(x)$. (We write $\lambda(x)$ if $\lambda_{+}(x)=\lambda_{-}(x)$.) However, there are instances where a pointwise Lyapunov exponent is different from the Lyapunov exponent of all invariant measures. This is shown in Proposition 3 .

\section{Proof of Theorem 1}

Lemma 1. If $\mu \in \mathcal{M}$ and $\lambda(\mu)<0$, then $\mu$ is the Dirac- $\delta$ measure equidistributed on an attracting periodic orbit.

Proof. This follows from Przytycki's result 15] which states that for $\mu \in \mathcal{M}, \lambda(x) \geq$ 0 for $\mu$-a.e. $x$.

For the proof of Theorem 1, we need a construction developed by Hofbauer [5], called canonical Markov extension. This Markov system is $(\hat{I}, \hat{f})$, where $X$ is a disjoint union of closed intervals. Let $\mathcal{P}=\mathcal{P}_{0}=\left\{\xi_{0}, \ldots, \xi_{r}\right\}$ be the partition of $I$ into the monotonicity intervals of $f$. Also write $\mathcal{P}_{n}=\bigvee_{i=0}^{n-1} f^{-i}\left(\mathcal{P}_{0}\right)$, and $\mathcal{P}_{n}[x]$ is the element of $\mathcal{P}_{n}$ containing $x$. We will construct $X$ inductively.

- The base $B:=I$ belongs to $\hat{I}$.

- If $D \in \hat{I}$, let $E=\overline{f\left(D \cap \xi_{i}\right)}$. If the interval $E$ is equal to some already existing $D^{\prime} \in \hat{I}$, then define $\hat{f}(x, D)=\left(f(x), D^{\prime}\right)$. Otherwise, add $E$ disjointly to $\hat{I}$ and let $\hat{f}(x, D)=(f(x), E)$. (Note that if $x \in \partial \xi_{i}$, then use $f(x)=\lim _{\xi_{i} \ni y \rightarrow x} T(y)$ to define $f$ on $\partial \xi$.)

The system $(\hat{I}, \hat{f})$ is Markov in the sense that any component $D$ of $\hat{I}$ equals some union of components of $\hat{I}$. If we define the projection by $\pi(x, D)=x$, then $\hat{f} \circ \pi=$ $\pi \circ f$. Due to the Markov property, the following is true:

$$
f^{n}\left(\mathcal{P}_{n}[x]\right)=D \in \hat{I} \text { if and only if } \hat{f}^{n}\left(\pi^{-1}(x) \cap B\right) \in D .
$$

If $\mu$ is $f$-invariant, then we can construct a measure $\hat{\mu}$ as follows: Let $\hat{\mu}_{0}$ be the measure $\nu$ lifted to the base $B$ and set $\hat{\mu}_{n}=\frac{1}{n+1} \sum_{i=0}^{n} \hat{\mu}_{0} \circ \hat{f}^{-i}$. Clearly $\mu=\hat{\mu}_{n} \circ \pi^{-1}$ for each $n$. As was shown in [6], $\hat{\mu}_{n}$ converges vaguely (i.e. on compact sets) to a limit measure, say $\hat{\mu}$. If $\mu$ is ergodic, then $\hat{\mu}$ is either a probability measure on $\hat{I}$, in which case we call $\mu$ liftable, or $\hat{\mu}(D)=0$ for all $D \in \hat{I}$.

Let us say that an $n$-periodic point $p$ with multiplier $\left|D f^{n}(p)\right| \leq 1$ is essential if it is (one-sided) attracting and there exists $p^{\prime} \in \operatorname{orb}(p)$ and a critical or boundary point $c$ such that $f^{i}\left(\left(c, p^{\prime}\right)\right) \cap \mathcal{C}=\emptyset$ for all $i \geq 0$. This applies for example to periodic points of multimodal maps with negative Schwarzian derivative. 
Proposition 2. Let $f$ be a $C^{3}$ multimodal interval map with non-flat critical points. Let $\mu$ be an ergodic invariant probability measure such that $\mu(p)=0$ for each periodic point $p$ that is (i) inessential with multiplier $\leq 1$, or (ii) belongs to the boundary of the basin of another periodic point. Then $\mu$ is liftable if and only if $\mu$ has a positive Lyapunov exponent.

This result was proved in 3 for unimodal maps with negative Schwarzian derivative. Here we give the details for the multimodal case, although the idea of proof is the same.

Proof. The "if" part is proved in [6] using a construction from 9], except that [6] does not cover the case of atomic measures. So let us assume that $\mu$ is the equidistribution of a hyperbolic repelling periodic orbit $\operatorname{orb}(p)$, and that $p$ is not a boundary point of the basin of a periodic attractor. Let $N=2 \times$ the period of $p$, so $f^{N}$ is orientation preserving in a neighbourhood of $p$. Let $Z_{k}$ be the largest neighbourhood of $p$ on which $f^{k N}$ is monotone. Write $Z_{k}=\left(a_{k}, b_{k}\right) 2$ Because $p$ is not a boundary point of the basin of a periodic attractor, $a_{k}$ and $b_{k}$ are precritical points. More precisely, there are $n_{a}, n_{b}<N$ such that $f^{n_{a}}\left(a_{1}\right), f^{n_{b}}\left(b_{1}\right) \in \mathcal{C}$, and because $f^{N}\left(Z_{k+1}\right)=Z_{k}, a_{k} \in f^{-n_{a}-(k-1) N}(\mathcal{C})$ and $b_{k} \in f^{-n_{b}-(k-1) N}(\mathcal{C})$. It follows that if $p_{0}=\pi^{-1}(p) \cap B$, then $\hat{f}^{i k}\left(p_{0}\right) \in \pi^{-1}(p) \cap K_{N}$, where $K_{N}$ is the compact part of $\hat{I}$ consisting of all components $D$ that can be reached by a path $B \rightarrow D_{1} \rightarrow \cdots \rightarrow D$ of length $\leq N$. Clearly $\pi^{-1}(p) \cap K_{N}$ is finite, so it contains an $N$-periodic point $\hat{p}$. Because the lift $\hat{\mu}$ of $\mu$ is unique (see $[\underline{6}$ ), $\hat{\mu}$ must be equal to the equidistribution on $\operatorname{orb}(\hat{p})$.

For the "only if" part let us start proving that the equidistribution on a stable or neutral periodic orbit is non-liftable. Since such an orbit is essential, there is a point $p$ in this orbit and a critical (or boundary) point $c$ such that $f^{n}((c, p)) \cap \mathcal{C}=\emptyset$ for all $n \geq 0$. Assume that $p \in \xi_{k} \in \mathcal{P}$, and let $\hat{p}=\pi^{-1}(p) \cap B$ be the lift of $p$ to the base $B$ of the Markov extension. Then $\hat{f}(\hat{p})$ belongs to a successor $D:=f\left(\xi_{k}\right)$ of $B$, and $f(c) \in \partial D$. But since $f^{n}((c, p))$ never intersects a critical point, each $\hat{f}^{n}(\hat{p})$ belongs a different component of $\hat{I}$. Therefore, the limit of the measures $\hat{\mu}_{n}$ is not liftable.

Let us assume that $\mu$ is liftable, $\hat{\mu}$ being the lifted measure. We will show that $\lambda(\mu)>0$. Let $D \in \hat{I}$ be such that $\hat{\mu}(D)>0$ and let $J$ be an interval, compactly contained in $D$, such that $\hat{\mu}(J)>0$. Since $\mu$ is not the equidistribution on the orbit of a stable or neutral periodic point $p, \pi(\bar{J})$ can be chosen disjoint from $\operatorname{orb}(p)$. Moreover we can choose $J$ such that $\operatorname{orb}(\partial J) \cap J=\emptyset$. Let $\hat{F}: J \rightarrow J$ be the first return map to $J$. By our conditions on $J$ each branch $\hat{F}: J_{i} \rightarrow J$ of $\hat{F}$ is onto, and by the Markov property of $\hat{f},\left.\hat{F}\right|_{J_{i}}$ is extendible monotonically to a branch that covers $D$. Clearly each branch of $\hat{F}$, say $\left.\hat{F}\right|_{J_{i}}=\left.\hat{f}^{s}\right|_{J_{i}}$, contains an $s$-periodic point $q$. Due to a result by Martens, de Melo and van Strien [11] and also [12, Theorem IV B'], there exists $\varepsilon>0$ such that $\left|\left(\hat{f}^{s}\right)^{\prime}(q)\right|>1+\varepsilon$, independent of the branch. If $J$ is sufficiently small, the Koebe Principle [12, Section IV.1] yields that $\left|\hat{F}^{\prime}(x)\right|>1+\frac{\varepsilon}{2}$ for all $x \in J$. The Koebe Principle holds for maps with negative Schwarzian derivative, but the work of Kozlovski 8 and in the multimodal setting van Strien \& Vargas [17] implies that the branches of $F$ have negative Schwarzian

\footnotetext{
${ }^{2}$ If $p$ is a boundary point of the interval $I$, then we have to adjust this argument to one-sided neighbourhoods $\left(a_{k}, p\right]$ or $\left[p, b_{k}\right)$.
} 
derivative if $f$ is $C^{3}$ and $J$ sufficiently small. It is at this moment that we use the $C^{3}$ assumption. It follows from the thesis of Mike Todd [18, that a $C^{2+\varepsilon}$ assumption suffices for unimodal maps.

Clearly $\frac{\hat{\mu}}{\hat{\mu}(J)}$ is an $\hat{F}$-invariant probability measure on $J$. Let $J_{i}, i \in \mathbb{N}$, be the branch-domains of $\hat{F}$, and let $s_{i}$ be such that $\left.\hat{F}\right|_{J_{i}}=\left.\hat{f}^{s_{i}}\right|_{J_{i}}$. Since we can write $\hat{\mu}$ as

$$
\int \varphi d \hat{\mu}=\sum_{i} \sum_{j=0}^{s_{i}-1} \int_{J_{i}} \varphi \circ \hat{f}^{j} d \hat{\mu}
$$

we get

$$
\begin{aligned}
\lambda(\hat{\mu}) & =\sum_{i} \sum_{j=0}^{s_{i}-1} \int_{J_{i}} \log \left|\hat{f}^{\prime}\right| \circ \hat{f}^{j} d \hat{\mu} \\
& =\sum_{i} \int_{J_{i}} \log \left|\left(\hat{f}^{s_{i}}\right)^{\prime}\right| d \hat{\mu}=\sum_{i} \int_{J_{i}} \log \left|\hat{F}^{\prime}\right| d \hat{\mu} \\
& \geq \sum_{i} \hat{\mu}\left(J_{i}\right) \log \left(1+\frac{\varepsilon}{2}\right)=\hat{\mu}(J) \log \left(1+\frac{\varepsilon}{2}\right)>0 .
\end{aligned}
$$

Because $f^{\prime}(\pi(x))=\hat{f}^{\prime}(x)$ for all $x \in \hat{I}$, this concludes the proof.

Now we are ready to prove Theorem 1

Proof. First assume that $\lambda(\mu)>0$, and let $\hat{\mu}$ be its lift to the Markov extension. Assume that $g: J \rightarrow J$ has lift $\hat{g}: \hat{J} \rightarrow \hat{J}$. Define $\hat{h}: \hat{I} \rightarrow \hat{J}$ as $\hat{h} \mid\left(D_{n} \subset \hat{I}\right)=$ $h \mid\left(D_{n} \subset I\right)$. Then $\hat{h}^{*} \hat{\mu}$ is a $\hat{g}$-invariant probability measure with $h^{*} \mu=\hat{h}^{*} \hat{\mu} \circ \pi^{-1}$. Thus $\hat{h}^{*} \hat{\mu}$ is the lift of $h^{*} \mu$. It follows that $h^{*} \mu$ is liftable and hence has a positive Lyapunov exponent. (Here we should recall that $\mu$ and $h^{*} \mu$ are non-atomic, so they are not associated with any periodic orbit, essential or not.)

By Lemma 1, negative Lyapunov exponents can only occur for atomic measures. Hence the remaining case $\lambda(\mu)=0$ is also preserved under conjugacy.

\section{Pointwise Lyapunov exponents}

Proof of Proposition 1. We give a counterexample based on the unimodal maps $f(x)=4 x(1-x)$ and $g(x)=\sin (\pi x)$, both having negative Schwarzian derivative. These maps are conjugate on the unit interval. Due to the well-known smooth conjugacy with the tent map, we have that $\lambda(x)=\log 2$ whenever the limit exists and $f^{n}(x) \neq 1$ for all $n \geq 1$. However, the limit need not always exist. Indeed, let $\left(n_{k}\right)$ be a superexponentially increasing integer sequence, and $y \in[0,1]$ a point such that:

- $y_{i} \in\left[\frac{1}{2}, 1\right]$ for $y_{i}=f^{i}(y)$ and $0<i<n_{1}$. Assuming $n_{1}$ is large, this means that $y_{i} \approx p=\frac{3}{4}$, the fixed point of $f$, and hence $D f^{n_{1}}(y) \approx 2^{n_{1}}$.

- $y_{n_{1}}$ is close to $c$ such that $y_{n_{1}} \approx 1$ and $y_{i} \in\left[0, \frac{1}{2}\right]$ for $n_{1}+1<i \leq$ $2.1 n_{1}$. This means that $y_{i} \approx 0$, the other fixed point, and since $f^{\prime}(0)=$ 4 , we obtain that $\left|0-y_{n_{1}+2}\right|=\mathcal{O}\left(4^{-1.1 n_{1}}\right)$. Consequently, $\left|c-y_{n_{1}}\right|=$ $\mathcal{O}\left(\sqrt{4^{-1.1 n_{1}}}\right)=\mathcal{O}\left(2^{-1.1 n_{1}}\right)$, and hence $\left|D f^{1+n_{1}}(y)\right|=\mathcal{O}\left(2^{n_{1}} \cdot 2^{-1.1 n_{1}}\right)=$ $\mathcal{O}\left(2^{-0.1 n_{1}}\right)$, whereas $\left|D f^{2.1 n_{1}}(y)\right|=\mathcal{O}\left(2^{n_{1}} \cdot 2^{-0.1 n_{1}} \cdot 4^{1.1 n_{1}}\right)=\mathcal{O}\left(2^{2.1 n_{1}}\right)$.

- Let $y_{i} \in\left[\frac{1}{2}, 1\right]$ for $2.1 n_{1}<i<n_{2}$. Hence, we find $D f^{n_{2}}(y)=\mathcal{O}\left(2^{n_{2}}\right)$. 
- $y_{n_{2}}$ is close to $c$ (and hence $y_{1+n_{2}}$ close to 1 ) such that $y_{i} \in\left[0, \frac{1}{2}\right]$ for $n_{2}+1<$ $i \leq 2.1 n_{2}$. It follows that $\left|D f^{1+n_{2}}(y)\right|=\mathcal{O}\left(2^{n_{1}} \cdot 2^{-1.1 n_{2}}\right)=\mathcal{O}\left(2^{-0.1 n_{2}}\right)$ and $\left|D f^{2.1 n_{1}}(y)\right|=\mathcal{O}\left(2^{2.1 n_{2}}\right)$.

Continue in this fashion, and we find that the lower Lyapunov exponent is $\underline{\lambda}(y)=$ $\lim \inf \frac{1}{n} \log D f^{n}(y)=-0.1 \log 2$, whereas the upper Lyapunov exponent is $\overline{\bar{\lambda}}(y)=$ $\limsup \frac{1}{n} \log D f^{n}(y)=\log 2$.

Now to do the same for $g=h \circ f \circ h^{-1}$ and the corresponding $\tilde{y}=h(y)$, we have to deal with different multipliers: $|D g(0)|=\pi<|D f(0)|$ and $\alpha:=|D g(\tilde{p})| \approx$ $2.12>|D f(p)|$ for $\tilde{p}=h(p)$. We now get that $\left|D g^{1+n_{k}}(\tilde{y})\right|=\mathcal{O}\left(\left(\frac{\alpha}{\pi^{0.55}}\right)^{n_{k}}\right)$ is still exponentially large, so in this case, $\underline{\lambda}(\tilde{y})>0$.

3.1. Example. We want to compare the results in this paper to an example from 2]. In this example, two conjugate smooth unimodal maps $f_{1}$ and $f_{2}$ (in fact, $f_{1}$ is quadratic and $f_{2}$ is a sine function), for which

$$
\inf _{\varepsilon>0} \lim _{n \rightarrow \infty} \frac{1}{n}\left\{0 \leq i<n: f_{k}^{i}(c) \in(p-\varepsilon, p+\varepsilon)\right\}=1
$$

for $k=1,2$, and $p=p_{k}$ is the orientation reversing fixed point of $f_{k}$. Yet $f_{1}$ has an acip (i.e. an absolutely continuous (w.r.t Lebesgue) invariant probability measure), and $f_{2}$ has not. Clearly the Dirac measure $\delta_{p}$ is the only weak limit point of $\left(\frac{1}{n} \sum_{i=0}^{n-1} \delta_{f_{k}^{i}(c)}\right)$ for $k=1,2$. Any non-liftable measure belongs to the convex hull of weak accumulation points of $\left(\frac{1}{n} \sum_{i=0}^{n-1} \delta_{f^{i}(c)}\right)$; see 6 . Consequently, $f_{k}$ has only liftable invariant measures, all of which have positive Lyapunov exponents. The acip of $f_{1}$ does not transform under $h^{*}$ to an acip of $f_{2}$, and in fact, there is not a single $f$-invariant measure $\mu$ such that $h^{*} \mu$ is absolutely continuous.

A result by Keller [7] implies that for $k=2, \delta_{p}$ is the only weak limit point of $\left(\frac{1}{n} \sum_{i=0}^{n-1} \delta_{f_{2}^{i}(x)}\right)$ for Lebesgue-a.e. $x$. Recall that a physical measure $\mu$ is defined by the fact that for every continuous observable $\varphi:[0,1] \rightarrow \mathbb{R}$,

$$
\mu(\varphi):=\int \varphi d \mu=\lim _{n \rightarrow \infty} \frac{1}{n} \sum_{i=0}^{n-1} \varphi \circ f^{i}(x) \text { Lebesgue-a.e. }
$$

Therefore $\delta_{p}$ is the physical measure of $f_{2}$. However $\liminf \operatorname{in}_{n} \frac{1}{n} \log \left|D f^{n}(x)\right|=0$ Lebesgue-a.e., because otherwise there would be an acip by [7. This shows that it is important in (1) to have continuous, not just $L^{1}$, observables.

Since $f_{k}$ is not Collet-Eckmann, $\inf \left\{\lambda(\mu): \mu\right.$ is $f_{k}$-invariant $\}=0$ for $k=1,2$. Therefore the infimum of Lyapunov exponents is not attained. This is in contrast to the Lyapunov exponent of invariant measures supported on hyperbolic sets; see 4]. The results below show that the spectrum of pointwise Lyapunov exponents can be strictly larger than the spectrum of Lyapunov exponents of measures.

Proposition 3. There exists a unimodal map $f$ such that $\lambda(\mu)>0$ for every $\mu \in \mathcal{M}$, but there is a point $x$ whose Lyapunov exponent exists (as a limit) and equals 0 .

Proof. We start by introducing some notation for unimodal maps. A point $z<c$ is called a precritical point closest to $c$ if $f^{S}(z)=c$ for some iterate $S$ and $f^{i}(c, z) \not \supset c$ for $0 \leq i \leq S$. There is an increasing sequence $\left(z_{k}\right)$ of such precritical points, starting with $z_{0} \in f^{-1}(c)$. The corresponding iterates $S_{k}$ such that $f^{S_{k}}\left(z_{k}\right)=0$ are called cutting times. Clearly $S_{0}=1$ and $S_{k}>S_{k-1}$ for each $k \geq 1$. Let 
$U_{k}=\left(z_{k}, z_{k+1}\right)$ and $\hat{U}_{k}=\left(\hat{z}_{k+1}, \hat{z}_{k}\right)$ be the interval at the other side of $c$ such that $f\left(U_{k}\right)=f\left(\hat{U}_{k}\right)$. Note that the intervals $\left(z_{k-1}, c\right)$ and $\left(c, \hat{z}_{k-1}\right)$ are the largest intervals adjacent to $c$ on which $f^{S_{k}}$ is a diffeomorphism.

If $f$ has no periodic attractor, then $z_{k} \rightarrow c$. If there is a $b$-periodic attractor and $B$ is the component of its basin of attraction containing $c$, then $z_{k} \rightarrow \partial B$. In fact, if $f$ has a neutrally attracting periodic orbit (at a saddle node bifurcation), then $\partial B$ contains a point of this orbit, and $z_{k}$ converges to $\partial B$ in a polynomial way (the precise rate of convergence depends on degeneracy of the neutral periodic orbit).

In 22 this phenomenon is exploited by creating a cascade of almost saddle node bifurcations; there is an infinite sequence of integers $b_{n}$ and a map $f$ created as the limit of a sequence of maps $f_{n}$, where $f_{n}$ has a $b_{n}$-periodic orbit at a saddle node bifurcation. While perturbing $f_{n}$ to $f_{n+1}$, the geometric properties of the sequence $\left(z_{k}\right)$ is preserved to some extent. In the example constructed in [2], the geometry of $\left(z_{k}\right)$ is such that $\left|z_{k}-z_{k+1}\right|$ decreases polynomially for values of $k$ associated to almost saddle node bifurcations, and $\left|z_{k}-z_{k+1}\right|$ decreases exponentially for other values of $k$. One can construct examples where the first behaviour dominates, so that the following properties hold:

(1) $1 \leq S_{k}-S_{k-1} \leq 2$ for all $k \geq 1$; hence $k<S_{k} \leq 2 k$ for $k \geq 1$.

(2) The distances $\left|f^{S_{k}}(c)-f^{S_{k}}\left(z_{k+1}\right)\right|,\left|f^{S_{k}}\left(z_{k+1}\right)-f^{S_{k}}\left(z_{k}\right)\right|$ and $\mid f^{S_{k}}\left(z_{k}\right)-$ $f^{S_{k}}\left(z_{k-1}\right) \mid$ are bounded away from 0 , uniformly in $k$. Using the Koebe Principle [12, we conclude that the distortion of $f^{S_{k}} \mid U_{k}$ and $f^{S_{k}} \mid \hat{U}_{k}$ is uniformly bounded.

(3) $\lim _{k} \frac{1}{k} \log \left|z_{k}-z_{k+1}\right|^{-1}=0$.

Construct the induced map $F$ by $F \mid U_{k} \cup \hat{U}_{k}=f^{S_{k}}$. It is easy to verify from property (1) that $F\left(U_{k}\right)=F\left(\hat{U}_{k}\right)=\left(z_{0}, c\right),\left(z_{1}, c\right),\left(c, \hat{z}_{0}\right)$ or $\left(c, \hat{z}_{1}\right)$. Hence $F$ is a Markov map. For any $x$, write $\chi_{n}(x)=k$ if $F^{n}(x) \in U_{k} \cap \hat{U}_{k}$. Also, let $t_{n}=\sum_{i=0}^{n-1} S_{\chi_{i}(x)}$, so $x_{n}:=F^{n}(x)=f^{t_{n}}(x)$. Because of the Markov properties of $F$, there are points $x$ such that $\chi_{n}(x) \rightarrow \infty$ so slowly that $\frac{t_{n+1}-t_{n}}{t_{n}} \rightarrow 0$. Therefore

$$
\begin{aligned}
\frac{1}{t_{n}} \log \left|D f^{t_{n}}(x)\right| & =\frac{1}{t_{n}} \log \prod_{i=0}^{n-1}\left|D f^{S_{\chi_{i}}}\left(x_{i}\right)\right| \\
& =\frac{\sum_{i=0}^{n-1} \log \left|D f^{S}{\chi_{\chi_{i}}}\left(x_{i}\right)\right|}{\sum_{i=0}^{n-1} S_{\chi_{i}}} \\
& \sim \frac{K \sum_{i=0}^{n-1} \log \left|z_{\chi_{i}}-z_{\chi_{i}+1}\right|^{-1}}{\sum_{i=0}^{n-1} \chi_{i}} \rightarrow 0,
\end{aligned}
$$

by property (3). Here $K$ depends only on the image-length and distortion of the branches of $F$, which are uniform by property (2). Finally, for intermediate values of $t$, i.e. $t_{n} \leq t<t_{n+1}$, we have

$$
L^{t-t_{n+1}}\left|D f^{t_{n+1}}(x)\right| \leq\left|D f^{t}(x)\right| \leq L^{t-t_{n}}\left|D f^{t_{n}}(x)\right|
$$

for $L=\sup |D f|<\infty$. By the assumption that $\frac{t_{n+1}-t_{n}}{t_{n}} \rightarrow 0$, we obtain

$$
\lim _{t} \frac{1}{t} \log \left|D f^{t}(x)\right|=0
$$

as well. This concludes the proof. 


\section{REFERENCES}

[1] A. Blokh, Decomposition of dynamical systems on an interval, Russian Math. Surveys 38 (1983) 133-134. MR0718829 (86d:54060)

[2] H. Bruin, The existence of absolutely continuous invariant measures is not a topological invariant for unimodal maps, Ergod. Th. and Dynam. Sys. 18 (1998) 555-565. MR1631716 (99i:58090)

[3] H. Bruin, G. Keller, Equilibrium states for S-unimodal maps, Ergod. Th. and Dynam. Sys. 18 (1998) 765-789. MR:1645373 (2000g:37039)

[4] Yongluo Cao, Stefano Luzzatto, Isabel Rios, Minimum principle for Lyapunov exponents and a higher-dimensional version of a Theorem of Mañé, to appear in Qual. Th. of Dyn. Sys.

[5] F. Hofbauer, On intrinsic ergodicity of piecewise monotonic transformations with positive entropy, Israel J. of Math. 34 (1979) 213-237. MR0570882 (82c:28039a)

[6] G. Keller, Lifting measures to Markov extensions, Monatsh. Math. 108 (1989) 183-200. MR.1026617 (91b:28011)

[7] G. Keller, Exponents, attractors and Hopf decompositions for interval maps, Ergod. Th. and Dynam. Sys. 10 (1990) 717-744. MR1091423 (92e:58118)

[8] O. Kozlovski, Getting rid of the negative Schwarzian derivative condition, Ann. Math. 152 (2000) 743-762. MR1815700 (2002e:37050)

[9] F. Ledrappier, Some properties of absolutely continuous invariant measures on an interval, Ergod. Th. and Dynam. Sys. 1 (1981) 77-93. MR0627788 (82k:28018)

[10] S. Luzzatto, L. Wang, Topological invariance of generic non-uniformly expanding multimodal maps, Preprint (2003).

[11] M. Martens, W. de Melo, S. van Strien, Julia-Fatou-Sullivan theory for real one-dimensional dynamics, Acta Math. 168 (1992) 273-318. MR.1161268 (93d:58137)

[12] W. de Melo, S. van Strien, One-Dimensional Dynamics, Springer, Berlin, Heidelberg, New York (1993). MR.1239171 (95a:58035)

[13] T. Nowicki, F. Przytycki, Topological invariance of the Collet-Eckmann property for Sunimodal maps, Fund. Math. 155 (1998) 33-43. MR1487986 (99a:58058)

[14] T. Nowicki, D. Sands, Non-uniform hyperbolicity and universal bounds for $S$-unimodal maps, Invent. Math. 132 (1998) 633-680. MR1625708 (99c:58122)

[15] F. Przytycki, Lyapunov characteristic exponents are nonnegative, Proc. Amer. Math. Soc. 119 (1993) 309-317. MR.1186141 (93k:58193)

[16] F. Przytycki, J. Rivera-Letelier, S. Smirnov, Equivalence and topological invariance of conditions for non-uniform hyperbolicity in the iteration of rational maps, Invent. Math. 151 (2003) 29-63. MR.1943741 (2003k:37065)

[17] S. van Strien, E. Vargas, Real bounds, ergodicity and negative Schwarzian for multimodal maps, J. Amer. Math. Soc. 17 (2004) 749-782. MR2083467

[18] M. Todd, One-dimensional dynamics: cross-ratios, negative Schwarzian and structural stability, Ph.D. thesis Warwick (2004).

Department of Mathematics, University of Surrey, Guildford, Surrey GU2 7XH, UNITED KINGDOM

E-mail address: h.bruin@eim.surrey.ac.uk

URL: http://www.maths.surrey.ac.uk/showstaff?H.Bruin

Department of Mathematics, Imperial College, London SW7 2AZ, United Kingdom

E-mail address: stefano.luzzatto@imperial.ac.uk

$U R L:$ http://www.ma.ic.ac.uk/ luzzatto 